\title{
Steady Flow of Couple-Stress Fluid in Constricted Tapered Artery: Effects of Transverse Magnetic Field, Moving Catheter, and Slip Velocity
}

\author{
Hamzah Bakhti ${ }^{1}$ and Lahcen Azrar ${ }^{1,2}$ \\ ${ }^{1}$ MMC Team, Department of Mathematics, Faculty of Sciences \& Techniques, AEU, BP 416 Tangier, Morocco \\ ${ }^{2}$ MMC Team, Department of Mechanical Engineering, Faculty of Engineering, KAU, Jeddah, Saudi Arabia
}

Correspondence should be addressed to Hamzah Bakhti; bakhti_hamzah@outlook.com

Received 9 July 2015; Revised 22 August 2015; Accepted 24 August 2015

Academic Editor: Taha Sochi

Copyright (C) 2016 H. Bakhti and L. Azrar. This is an open access article distributed under the Creative Commons Attribution License, which permits unrestricted use, distribution, and reproduction in any medium, provided the original work is properly cited.

\begin{abstract}
Steady flow of a couple-stress fluid in constricted tapered artery has been studied under the effects of transverse magnetic field, moving catheter, and slip velocity. With the help of Bessel's functions, analytic expressions for axial velocity, flow rate, impedance, and wall shear stress have been obtained. It is of interest to note that these solutions can be used for different types of fluid flow in tubes and not only the case of blood. The effects of various geometric parameters, the parameters arising out of the fluid considered and the magnetic field, are discussed by considering the slip velocity, the catheter velocity, and tapering angle. The study of the above model is very important as it has direct applications in the treatment of cardiovascular diseases.
\end{abstract}

\section{Introduction}

Catheters are semirigid, thin tubes made from medical grade materials serving a broad range of functions and can be inserted in the body to detect and identify diseases or perform a surgical procedure inside the heart, brain, arms, legs, or lungs [1]. But the problem is how we can know that the catheter is going the right way or where is it. Most hospitals use X-ray machine, mounted above the couch to check the catheter's progress. Low-dose X-rays are used to monitor the progress of the catheter tip. But if we want to navigate the catheter's passage in real time, some ten to twenty X-ray images are made every second. Even though the radiation dose involved is very low, no radiation at all is much healthier for both the patient and the medical staff who run the risk of being exposed to the radiation on daily basis. Researchers developed a magnetic navigation system for medical instruments such as catheters and guide wires. The catheters with magnetic tips are steered within the patient, without the need for an electrophysiologist to maneuver the catheter or guide wire placement manually. A magnetic sensor on the tip of the medical instrument measures an external magnetic field and reports exactly where the tip of the instrument is located [2].

Each year, heart disease is at the top of the list of the country's most serious health problems. Statistics show that cardiovascular disease in some countries, like America, is the first health problem and the leading cause of death (recent statistics released by the American Heart Association). Catheters are often used to treat stenosis (partial occlusion of the blood vessel), aneurysm (dilation of the blood vessel resulting in stretching of the vessel wall), and embolism (complete occlusion of a blood vessel by a blood clot or some other particle), Figure 1. The catheters may contain a balloon that can be used to stretch the blood vessel or a rinsing mechanism or laser device to remove embolism. They are also used to measure blood pressure in situ. Sometimes a catheter is used to deliberately create embolism, for example, to stop the flow of blood to a tumor in order to mortify it. An aneurysm can also be treated by means of embolisation [3].

The study of blood flow through different types of arteries is of considerable importance in many cardiovascular diseases; one of them is atherosclerosis. The blood flow through 


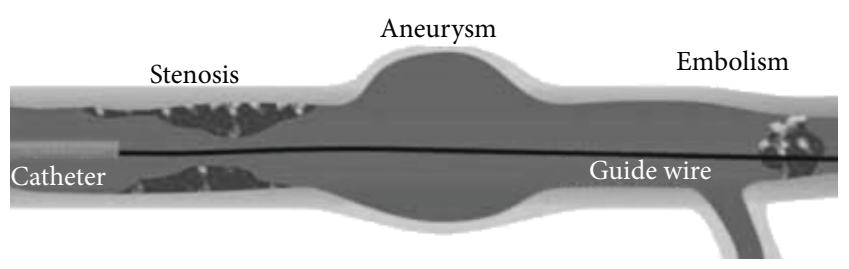

FIGURE 1: Catheters are used to treat stenosis (partial occlusion of the blood vessel), aneurysm (dilation of the blood vessel resulting in stretching of the vessel wall), and embolism (complete occlusion of a blood vessel by a blood clot or some other particle) (a reproduction from Delft Outlook 2004).

an artery has drawn the attention of researchers for a long time up to now, due to its great importance in medical sciences. Under normal conditions, blood flow in the human circulatory system depends upon the pumping action of the heart and this produces a pressure gradient throughout the arterial network.

Many researchers have studied blood flow in the artery by considering blood as either Newtonian or non-Newtonian fluids; since blood is a suspension of red cells in plasma, it behaves as a non-Newtonian fluid at low shear rate. In the stenosed condition, substantial reduction in the lumen of an artery results in size effects (ratio of haematocrit to vessel diameter), which influences flow characteristics significantly. To study the size effect in the fluid flow, Stokes [4], Eringen [5], and Cowin [6] have proposed continuum model. Microcontinuum structure of the fluid is also referred to as couplestress fluid which is proposed by Stokes. The foreground of couple-stress fluid is to introduce size dependent effects as mentioned above that is not present in other classical viscous fluids. Because of its mathematical simplicity, it has been widely used by number of researchers; Chaturani and Upadhya [7] have studied the pulsatile flow of couple-stress fluid through circular tubes. The Poiseuille flow of couplestress fluid has been examined by Chaturani and Rathod [8].

The application of Magnetohydrodynamics in physiological problems is of growing interest and it is very important from both theoretical and practical points of view. The application of Magnetohydrodynamics in physiological problems is of growing interest. The flow of blood can be controlled by applying appropriate quantity of magnetic field. Kollin [9] has coined the idea of electromagnetic field in the medical research for the first time in the year 1936. Korchevskii and Marochnik [10] have discussed the possibility of regulating the blood movement in human system by applying magnetic field. Rao and Deshikachar [11] have investigated the effect of transverse magnetic field in physiological type of flow, through a uniform circular pipe. Vardanyan [12] showed that the application of magnetic field reduces the speed of blood flow. A mathematical model for two-layer pulsatile flow of blood with microorganism in a uniform tube under low Reynolds number and magnetic effect has been studied by Rathod and Gayatri [13]. Thus, all these researchers have reported that the effect of magnetic field reduces the velocity of blood.
There are many treatments available for diagnosing and treating constricted vessels. Catheterization (thin, flexible tube) is one of them, in which balloon angioplasty is a specialized form of catheterization. These procedures are widely used in the medical field for treating the atherosclerosis. Insertion of the catheter in a tube creates an annular region between inner wall of the artery and outer wall of the catheter which influences the flow field such as pressure distribution and shear stress at the wall. In view of its immense importance, the effect of the catheter on physiological parameters was discussed by the researchers [14, 15].

The shapes of the stenosis in the above aforesaid studies have been considered to be radially symmetric or asymmetric. But while stenosis is maturing, it may grow up in series manner, overlapping with each other, and it would appear like x-shape. Riahi et al. [16] observed the pressure gradient force, flow velocity, impedance, and wall shear stress in the overlapping stenotic zone at the critical height and at the throats of such stenosis. Here they considered steady nature of flow. Srivastava and Mishra [17] explored the arterial blood flow through an overlapping stenosis by treating the blood as a Casson fluid. They figured impedance and shear stress for different stenosis heights. Chakravarty and Mandal [18] discussed the effects of the overlapping stenosis under low shear rate flow.

The presence of red cell slip at the vessel wall was recommended theoretically by Vand [19], experimentally by Bennett [20] and Nubar [21], Chaturani and Biswas [22], and so forth. They used slip velocity at the wall in their analysis. Lately, Ponalagusamy [23, 25] has developed mathematical models for blood flow through stenosed arterial segment, by taking a slip velocity condition at the constricted wall. Thus, it is very appropriate to consider slip velocity at the wall of the stenosed artery in blood flow modeling. The catheter is actually moving into the body, so it is recommended to consider a nonnull velocity at the catheter wall; in this paper, we consider that the catheter is moving in the $z$-axis with a fixed velocity.

In the present analysis a mathematical model for the steady blood flow through tapered stenosed artery under the influence of a moving catheter, slip velocity, and a magnetic field is presented by considering blood as a couple-stress fluid in a circular tube. It is assumed that the magnetic field along the radius of the pipe is present, no external electric field is imposed, and magnetic Reynolds number is very small. The motivation for studying this problem is to understand the blood flow in an artery under the effect of magnetic field alongside with the catheter inserted into the blood vessel also when the fatty plaques of cholesterol and artery clogging blood clots are formed in the lumen of the artery.

The main aim of this work is to study these phenomena, obtain analytic expressions for axial velocity and shear stress, and also study the effect of magnetic field (Hartmann number $H)$ and couple-stress parameter $(\beta)$ on the velocity and the effects of Hartmann number on the fluid velocity. Hence, the present mathematical model gives a simple form of velocity expression for the blood flow so that it will help not only people working in the field of physiological fluid dynamics but also the medical practitioners. 


\section{Problem Formulation}

Let us consider a two-dimensional steady flow of blood through a rigid tapered stenosed tube by considering blood as an electrically conducting, incompressible, couple-stress fluid. The magnetic field is acting along the radius of the tube. The magnetic Reynolds number of the flow is assumed to be sufficiently small that the induced magnetic and electric fields can be neglected [24]. The catheter is assumed to be moving in the $z$-axis direction.
Mathematical model of blood flow through a tapered stenosed arterial segment in the presence of a moving catheter and a magnetic field is to be built to study the impact of various geometric, Hartman, and fluid parameters on physiological parameters. The geometry of the tapered stenosed artery is shown in Figures 2 and 3 and is expressed mathematically with inputs from [26] as

$$
R(z)= \begin{cases}\left(R_{0}+\zeta z\right)\left(1-\frac{\epsilon n^{n /(n-1)}}{(n-1) L_{0}^{n}}\left(L_{0}^{n-1}(z-d)-(z-d)^{n}\right)\right), & d \leq z \leq d+L_{0} \\ R_{0}+\zeta z, & \text { otherwise }\end{cases}
$$

where $R_{0}$ is the radius of the annular region in case of nontapered artery in the nonconstricted domain, $R_{c}$ is the radius of the catheter, $L_{0}$ is the stenosis length, $d$ indicates the location of stenosis, $\epsilon$ is the maximum height of the stenosis into the lumen, and $\zeta=\tan \phi$ is the tapering parameter which represents the slope of the tapered vessel with $\phi$ being the tapering angle. $\phi<0, \phi>0$, and $\phi=0$ are for converging taper, diverging taper, and no-taper, respectively.

The current density $\mathbf{J}$ is expressed by

$$
\mathbf{J}=\sigma(\mathbf{E}+\mathbf{V} \times \mathbf{B}),
$$

where $\mathbf{E}$ is the electric field intensity, $\sigma$ is the electrical conductivity, $\mathbf{B}$ is the magnetic flux intensity, and $\mathbf{V}$ is the velocity vector. In the momentum equation, the electromagnetic force $\mathbf{F}_{m}$ is included and is defined as

$$
\mathbf{F}_{m}=\mathbf{J} \times \mathbf{B}=\sigma(\mathbf{E}+\mathbf{V} \times \mathbf{B}) \times \mathbf{B} .
$$

The conservation equations which govern the couplestress fluid flow including a Lorentz force can be written in the following form:

$$
\begin{aligned}
\nabla \cdot \mathbf{V}= & 0, \\
\rho(\mathbf{V} \cdot \nabla) \mathbf{V}= & -\nabla p+(\lambda+\mu) \nabla \nabla \cdot \mathbf{V}+\eta \Delta \nabla \nabla \cdot \mathbf{V} \\
& -\eta \Delta^{2} \mathbf{V}+\mu \Delta \mathbf{V}+\sigma(\mathbf{V} \times \mathbf{B}) \times \mathbf{B}+\rho \mathbf{f} \\
& +\frac{1}{2} \nabla \times(\rho \mathbf{l}),
\end{aligned}
$$

where $\Delta$ is the Laplacian operator. For couple-stress fluid, shear stress tensor is not symmetric. The force stress tensor $\boldsymbol{\tau}$ and the couple-stress tensor $\mathbf{M}$ that arises in the theory of couple-stress fluids are given by

$$
\begin{aligned}
\boldsymbol{\tau}= & (-p+\lambda \nabla \mathbf{V}) \mathbf{I}+\mu\left(\nabla \mathbf{V}+(\nabla \mathbf{V})^{T}\right)+\frac{1}{2} \mathbf{I} \\
& \times(\nabla \mathbf{M}+\rho \mathbf{l}), \\
\mathbf{M}= & m \mathbf{I}+2 \eta(\nabla(\nabla \times \mathbf{V}))+2 \eta^{\prime}(\nabla(\nabla \times \mathbf{V}))^{T},
\end{aligned}
$$

where $\rho$ is the density, $\mathbf{V}$ is velocity vector, $p$ is the pressure, $\lambda$ and $\mu$ are the viscosity coefficients, and $\eta$ and $\eta^{\prime}$ are couplestress coefficients. $\mathbf{f}$ is a body force and $\mathbf{l}$ is a body couple moment. Further, the materials constants $\mu, \lambda, \eta$, and $\eta^{\prime}$ satisfy the following inequalities:

$$
\begin{aligned}
\mu & \geq 0, \\
3 \lambda+2 \mu & \geq 0, \\
\eta & \geq 0, \\
\eta & \geq \eta^{\prime} .
\end{aligned}
$$

As the flow is steady and incompressible, in the absence of body force and body couple moment (4) reduce to

$$
\begin{aligned}
\nabla \cdot \mathbf{V} & =0, \\
\rho(\mathbf{V} \cdot \nabla) \mathbf{V} & =-\nabla p+\mu \Delta \mathbf{V}-\eta \Delta^{2} \mathbf{V}+\sigma(\mathbf{V} \times \mathbf{B}) \times \mathbf{B} .
\end{aligned}
$$

The flow is considered to take place under the influence of externally applied magnetic field in axial direction $z$. Under these assumptions, the governing equations may be written in the cylindrical coordinates system as follows.

Equation of continuity is

$$
\frac{\partial u}{\partial r}+\frac{u}{r}+\frac{\partial w}{\partial z}=0 .
$$

Equation of radial momentum is

$$
\begin{gathered}
\rho\left(u \frac{\partial u}{\partial r}+w \frac{\partial u}{\partial z}\right)=-\frac{\partial p}{\partial r}+\mu\left(\frac{\partial^{2} u}{\partial r^{2}}+\frac{1}{r} \frac{\partial u}{\partial r}-\frac{u}{r^{2}}\right. \\
\left.+\frac{\partial^{2} u}{\partial z^{2}}\right)-\eta\left(\frac{\partial^{4} u}{\partial r^{4}}+2 \frac{\partial^{4} u}{\partial r^{2} \partial z^{2}}+\frac{\partial^{4} u}{\partial z^{4}}+\frac{2}{r} \frac{\partial^{3} u}{\partial r^{3}}\right. \\
\left.+\frac{2}{r} \frac{\partial^{3} u}{\partial r \partial z^{2}}-\frac{3}{r^{2}} \frac{\partial^{2} u}{\partial r^{2}}-\frac{2}{r^{2}} \frac{\partial^{2} u}{\partial z^{2}}+\frac{3}{r^{3}} \frac{\partial u}{\partial r}-\frac{3}{r^{4}} u\right) .
\end{gathered}
$$




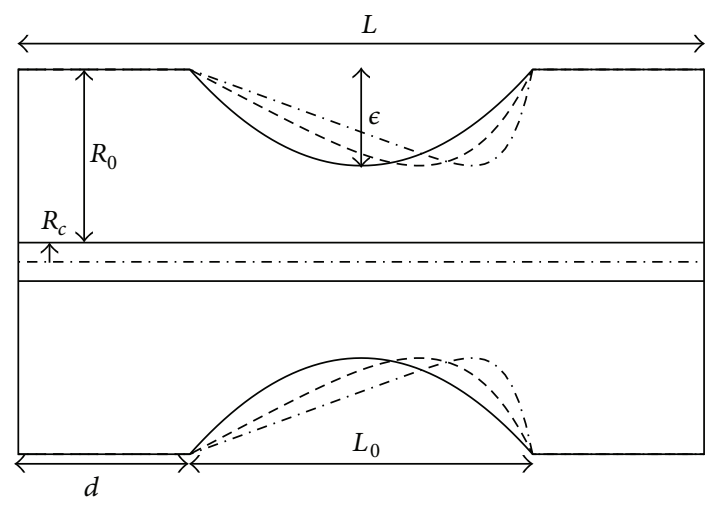

$-n=2$

- - $n=5$

-.. $n=15$

FIgURE 2: 2D view of a catheterized stenosed artery.

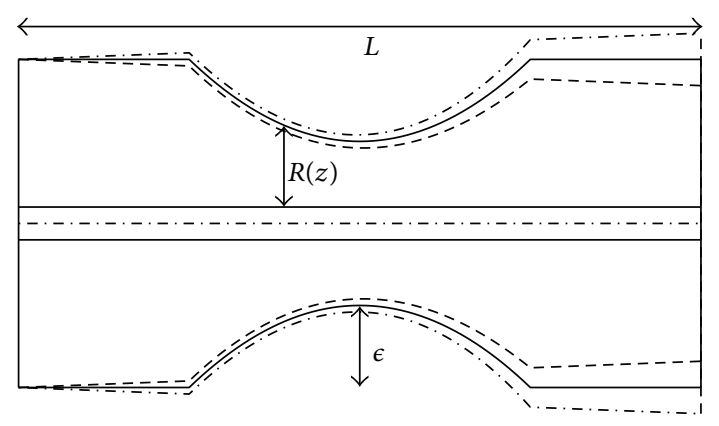

- $-\phi<0$

$-\phi=0$

..- $\phi>0$

FIGURE 3: 2D view of a tapered stenosed artery.
Equation of axial momentum is

$$
\begin{aligned}
& \rho\left(u \frac{\partial w}{\partial r}+w \frac{\partial w}{\partial z}\right)=-\frac{\partial p}{\partial z}+\mu\left(\frac{\partial^{2} w}{\partial r^{2}}+\frac{1}{r} \frac{\partial w}{\partial r}\right. \\
& \left.+\frac{\partial^{2} w}{\partial z^{2}}\right)-\eta\left(\frac{\partial^{4} w}{\partial r^{4}}+2 \frac{\partial^{4} w}{\partial r^{2} \partial z^{2}}+\frac{\partial^{4} w}{\partial z^{4}}+\frac{2}{r} \frac{\partial^{3} w}{\partial r^{3}}\right. \\
& \left.+\frac{2}{r} \frac{\partial^{3} w}{\partial r \partial z^{2}}-\frac{1}{r^{2}} \frac{\partial^{2} u}{\partial r^{2}}+\frac{1}{r^{3}} \frac{\partial w}{\partial r}\right)-\sigma B_{0}^{2} w
\end{aligned}
$$

where $B_{0}$ is the external transverse magnetic field. The nondimensional parameters are obtained as follows:

$$
\begin{aligned}
z^{*} & =\frac{z}{L_{0}}, \\
r^{*} & =\frac{r}{R_{0}}, \\
w^{*} & =\frac{w}{w_{0}}, \\
\epsilon^{*} & =\frac{\epsilon}{R_{0}}, \\
\zeta^{*} & =\frac{\zeta L_{0}}{R_{0}}, \\
u^{*} & =\frac{L_{0} u}{w_{0} R_{0}}, \\
p^{*} & =\frac{r_{0}^{2} p}{w_{0} L_{0} \mu},
\end{aligned}
$$

and then the dimensionless form of geometry (1) after dropping the stars is obtained as follows:

$$
R(z)= \begin{cases}(1+\zeta z)\left(1-\frac{\zeta n^{n /(n-1)}}{(n-1)}\left((z-\gamma)-(z-\gamma)^{n}\right)\right), & \gamma \leq z \leq \gamma+1 \\ 1+\zeta z, & \text { otherwise }\end{cases}
$$

where $\gamma=L_{0} / L_{1}$ and $w_{0}$ is a typical axial velocity.

Also, (8)-(10), on dropping the stars, become

$$
\begin{aligned}
& \frac{\partial u}{\partial r}+\frac{u}{r}+\frac{\partial w}{\partial z}=0 \\
& \operatorname{Re} \xi^{3}\left(u \frac{\partial u}{\partial r}+w \frac{\partial u}{\partial z}\right)=-\frac{\partial p}{\partial r}+\xi^{2}\left(\frac{\partial^{2} u}{\partial r^{2}}+\frac{1}{r} \frac{\partial u}{\partial r}-\frac{u}{r^{2}}\right. \\
& \left.+\xi^{2} \frac{\partial^{2} u}{\partial z^{2}}\right)-\frac{\xi^{2}}{\beta^{2}}\left[\frac{\partial^{4} u}{\partial r^{4}}+\frac{2}{r} \frac{\partial^{3} u}{\partial r^{3}}-\frac{3}{r^{2}} \frac{\partial^{2} u}{\partial r^{2}}+\frac{3}{r^{3}} \frac{\partial u}{\partial r}\right. \\
& \quad-\frac{3}{r^{4}} u
\end{aligned}
$$

$$
\begin{aligned}
& \left.+\xi^{2}\left(\frac{2}{r} \frac{\partial^{3} u}{\partial r \partial z^{2}}+2 \frac{\partial^{4} u}{\partial r^{2} \partial z^{2}}+\xi^{2} \frac{\partial^{4} u}{\partial z^{4}}-\frac{2}{r^{2}} \frac{\partial^{2} u}{\partial z^{2}}\right)\right] \\
& \operatorname{Re} \xi\left(u \frac{\partial w}{\partial r}+w \frac{\partial w}{\partial z}\right)=-\frac{\partial p}{\partial z}+\left(\frac{\partial^{2} w}{\partial r^{2}}+\frac{1}{r} \frac{\partial w}{\partial r}\right. \\
& \left.+\xi^{2} \frac{\partial^{2} w}{\partial z^{2}}\right)-\frac{1}{\beta^{2}}\left[\frac{\partial^{4} w}{\partial r^{4}}+\frac{2}{r} \frac{\partial^{3} w}{\partial r^{3}}-\frac{1}{r^{2}} \frac{\partial^{2} w}{\partial r^{2}}\right. \\
& \left.+\frac{1}{r^{3}} \frac{\partial w}{\partial r}+\xi^{4} \frac{\partial^{4} w}{\partial z^{4}}+2 \xi^{2}\left(\frac{\partial^{4} w}{\partial r^{2} \partial z^{2}}+\frac{1}{r} \frac{\partial^{3} w}{\partial r \partial z^{2}}\right)\right] \\
& -H^{2} w
\end{aligned}
$$


where $\xi=R_{0} / L_{0}, \operatorname{Re}=\rho u_{0} R_{0} / \mu$ is the Reynolds number, $\beta^{2}=R_{0}^{2} \mu / \eta$ is the couple-stress fluid parameter, and $H^{2}=$ $B_{0}^{2} R_{0}^{2} \sigma / \mu$ is the Hartmann number.

Under the assumption of mild stenosis that is $\epsilon / R_{0} \leq$ 1 and further assuming that $\xi=R_{0} / L_{0} \leq 1$, (13) get transformed into

$$
\begin{aligned}
\frac{\partial p}{\partial r}= & 0, \\
\frac{\partial p}{\partial z}= & \left(\frac{\partial^{2} w}{\partial r^{2}}+\frac{1}{r} \frac{\partial w}{\partial r}\right) \\
& -\frac{1}{\beta^{2}}\left(\frac{\partial^{4} w}{\partial r^{4}}+\frac{2}{r} \frac{\partial^{3} w}{\partial r^{3}}-\frac{1}{r^{2}} \frac{\partial^{2} w}{\partial r^{2}}+\frac{1}{r^{3}} \frac{\partial w}{\partial r}\right) \\
& -H^{2} w .
\end{aligned}
$$

Equation (15) can be written as

$$
\frac{\partial p}{\partial z}=\Delta w-\frac{1}{\beta^{2}} \Delta^{2} w-H^{2} w
$$

where

$$
\Delta=\frac{\partial^{2}}{\partial r^{2}}+\frac{1}{r} \frac{\partial}{\partial r} .
$$

It can be seen that the pressure variation depends only on the axial variable. The pressure gradient $\partial p / \partial z$ is produced by the pumping action of the heart.

The corresponding nondimensional boundary conditions are as shown below:

$$
\begin{aligned}
& w=v \quad \text { at } r=R(z), \\
& w=g \quad \text { at } r=R_{c} \text {, } \\
& \frac{\partial^{2} w}{\partial r^{2}}-\frac{\omega}{r} \frac{\partial w}{\partial r}=0 \quad \text { at } r=R(z), r=R_{c},
\end{aligned}
$$

where $v$ represents the slip velocity at the artery wall, $g$ is the velocity of the moving boundary (catheter), and $\omega=\eta^{\prime} / \eta$ is the parameter associated with the couple-stress fluid. No couple-stress effects will be present if $\eta^{\prime}=\eta$, which is equivalent to saying that the couple-stress tensor is symmetric.

\section{Solution of the Problem}

As a solution of (16) with the pressure gradient being taken as a constant, take

$$
-\frac{\partial p}{\partial z}=k_{s} .
$$

Let $n_{1}^{2}+n_{2}^{2}=\beta^{2}$ and $n_{1}^{2} n_{2}^{2}=\beta^{2} H^{2}$, which are given by

$$
\begin{aligned}
& n_{1}^{2}=\frac{\beta^{2}+\sqrt{\beta^{4}-4 \beta^{2} H^{2}}}{2}, \\
& n_{2}^{2}=\frac{\beta^{2}-\sqrt{\beta^{4}-4 \beta^{2} H^{2}}}{2} ;
\end{aligned}
$$

then, (16) is simplified to the form

$$
\left(\Delta-n_{1}^{2}\right)\left(\Delta-n_{2}^{2}\right) w=\beta^{2} k_{s} .
$$

The solution of the above equation is obtained as

$$
\begin{aligned}
w= & c_{1}(z) I_{0}\left(n_{1} r\right)+c_{2}(z) K_{0}\left(n_{1} r\right)+c_{3}(z) I_{0}\left(n_{2} r\right) \\
& +c_{4}(z) K_{0}\left(n_{2} r\right)+\frac{1}{H^{2}} k_{s},
\end{aligned}
$$

where $I_{0}$ and $K_{0}$ are modified Bessel function of order zero, first and second kinds, respectively. $c_{i}$ for $i=1,2,3,4$ are calculated numerically by solving the algebraic system obtained from the boundary conditions.

Volumetric flow rate $Q$, in steady flow through a tube, is obtained by integrating the velocity profile over a cross section of the tube. The nondimensional volumetric flow rate $Q$ across the radial distance is expressed as

$$
Q=\int_{R_{c}}^{R(z)} 2 r w d r
$$

which is obtained in the form $Q=-2 k_{s} F\left(R_{c}, R(z)\right)$, where

$$
\begin{aligned}
F\left(R_{c}, R(z)\right)= & \frac{d_{1}}{n_{1}}\left(R I_{1}\left(n_{1} R\right)-R_{c} I_{1}\left(n_{1} R_{c}\right)\right) \\
& +\frac{d_{2}}{n_{1}}\left(R_{c} K_{1}\left(n_{1} R_{c}\right)-R K_{1}\left(n_{1} R\right)\right) \\
& +\frac{d_{3}}{n_{2}}\left(R I_{1}\left(n_{2} R\right)-R_{c} I_{1}\left(n_{2} R_{c}\right)\right) \\
& +\frac{d_{4}}{n_{2}}\left(R_{c} K_{1}\left(n_{2} R_{c}\right)-R K_{1}\left(n_{2} R\right)\right) \\
& +\frac{R^{2}-R_{c}^{2}}{2 H^{2}},
\end{aligned}
$$

where $d_{i}=-c_{i} / k_{s}$, for $i=1,2,3,4$. The pressure drop $\Delta p$ across the stenosis between the sections $z=0$ and $z=L$ is obtained by

$$
\Delta p=\frac{Q}{2} \int_{0}^{L} \frac{1}{F\left(R_{c}, R(z)\right)} d z .
$$

The resistance to the flow (impedance) is obtained from

$$
\begin{aligned}
R_{f} & =\frac{\Delta p}{Q}=\frac{1}{2} \int_{0}^{L} \frac{1}{F\left(R_{c}, R(z)\right)} d z \\
& =\frac{1}{2}\left(\int_{0}^{d} \frac{1}{F\left(R_{c}, R(z)\right)} d z+\int_{d}^{d+L_{0}} \frac{1}{F\left(R_{c}, R(z)\right)} d z\right. \\
& \left.+\int_{d+L_{0}}^{L} \frac{1}{F\left(R_{c}, R(z)\right)} d z\right) .
\end{aligned}
$$

The dimensionless form of (20) is

$$
\begin{aligned}
R_{f} & =\frac{\Gamma}{2}\left(\int_{0}^{\gamma} \frac{1}{F\left(R_{c}, R(z)\right)} d z+\int_{\gamma}^{\gamma+1} \frac{1}{F\left(R_{c}, R(z)\right)} d z\right. \\
& \left.+\int_{\gamma+1}^{1 / \gamma} \frac{1}{F\left(R_{c}, R(z)\right)} d z\right),
\end{aligned}
$$

where $\Gamma=L_{0} / d$. 
The shear stress $\tau_{r z}$ is calculated using the expression

$$
\tau_{r z}=\tau_{r z}^{S}+\tau_{r z}^{A}
$$

where the symmetric and skew-symmetric parts of the stress are given by

$$
\begin{aligned}
\tau_{r z}^{S} & =\mu \frac{\partial w}{\partial r}, \\
\tau_{r z}^{A} & =\left(\frac{\partial m_{r \theta}}{\partial r}+\frac{m_{r \theta}+m_{\theta r}}{r}\right),
\end{aligned}
$$

respectively. Here,

$$
\begin{aligned}
& m_{r \theta}=\frac{1}{4}\left(\frac{\eta^{\prime}}{r} \frac{\partial w}{\partial r}-\eta \frac{\partial^{2} w}{\partial r^{2}}\right), \\
& m_{\theta r}=\frac{1}{4}\left(\frac{\eta}{r} \frac{\partial w}{\partial r}-\eta^{\prime} \frac{\partial^{2} w}{\partial r^{2}}\right) .
\end{aligned}
$$

Hence, the dimensionless shear stress for the artery is given by

$$
\begin{aligned}
& \tau_{r z} \\
& \begin{aligned}
= & n_{1}\left(c_{1} I_{1}\left(n_{1} r\right)-c_{2} K_{1}\left(n_{1} r\right)\right)\left(1-\frac{n_{1}^{2}}{4\left(n_{1}^{2}+n_{2}^{2}\right)}\right) \\
& +n_{2}\left(c_{3} I_{1}\left(n_{2} r\right)-c_{4} K_{1}\left(n_{2} r\right)\right)\left(1-\frac{n_{2}^{2}}{4\left(n_{1}^{2}+n_{2}^{2}\right)}\right) .
\end{aligned}
\end{aligned}
$$

Thus, the shear stress at the wall can be computed from (33) by taking $r=R(z)$.

\section{Results and Discussion}

The study of blood flow through catheterized stenosed tapered artery with the presence of a transverse magnetic field involves the integration of various geometric and fluid variables, which influences the physiological parameters such as the fluid velocity, rate flow, and wall shear stress. Closed form solutions are obtained in terms of modified Bessel's functions. The physiological dimensionless quantities such as the fluid velocity in the stenosis region and the wall shear stress at the maximum height of the stenosis are computed numerically for various values of the fluid and geometric parameters using the program MATLAB. The parameters considered are $n$ (shape parameter), $\zeta$ (tapered parameter), $R_{c}$ (catheter radius), $\Gamma$ (stenosis length), $\beta$ (couple-stress fluid parameter), $H$ (the Hartmann number), $v$ (slip velocity), and $g$ (catheter wall velocity). The obtained results are analyzed graphically. The results obtained in this study are in good agreement with those reported in the literature.

Figures 4-6 illustrate the variation of axial velocity profile for different values of $n, \zeta$, and $\beta$. It is observed from Figure 4 that the velocity profile increases for increasing the shape parameter and that actually this occurred due to the change of the stenosis height. It is observed from Figure 5 that the velocity increases by the increase in the tapered parameter

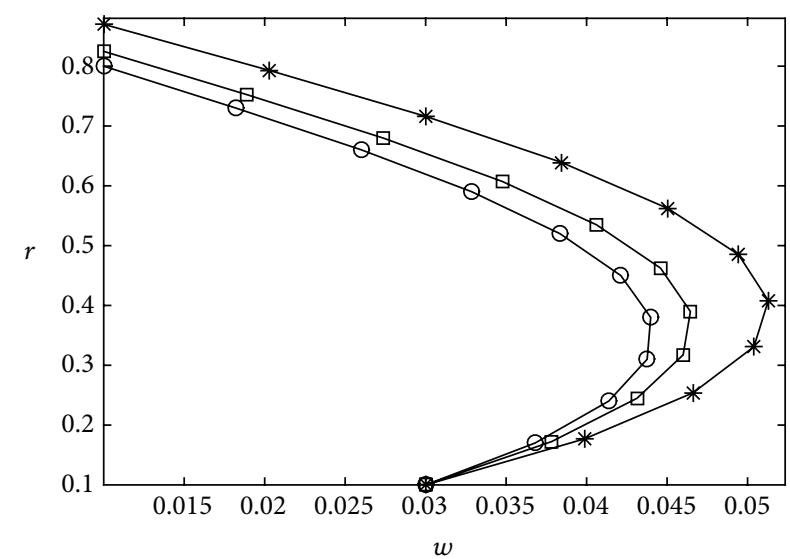

$$
\begin{aligned}
& \because n=2 \\
& \square n=5 \\
& \text { * } n=15
\end{aligned}
$$

FIGURE 4: Variation of axial velocity $w$ with respect to $n$ when $\zeta=0$, $\epsilon=0.2, \beta=5, H=1, v=0.01, g=0.03$, and $R_{c}=0.1$.

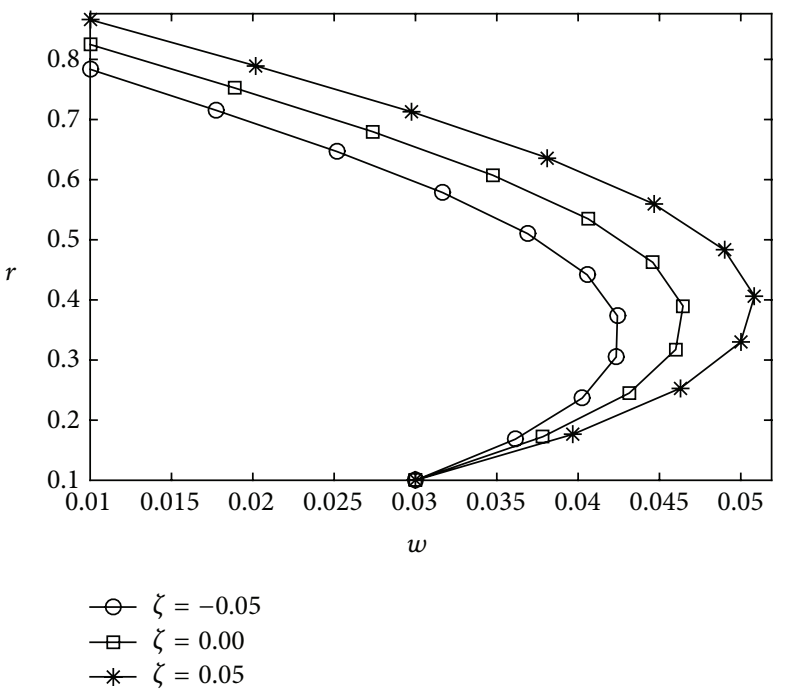

FIgURE 5: Variation of axial velocity $w$ with respect to $\zeta$ when $n=5$, $\epsilon=0.2, \beta=5, H=1, v=0.01, g=0.03$, and $R_{c}=0.1$.

$\zeta$ and the same goes for the couple-stress fluid parameter $\beta$, Figure 6 . It is to be noted as $\beta \rightarrow \infty$, the properties of couplestress in the fluid vanish and hence behave like a Newtonian fluid. Hence it is understood that the velocity is low in couplestress fluid when compared to that of Newtonian fluid.

In general, from Figure 7, it can be observed that the axial velocity is decreasing. The effect of the Hartmann number $H$ on the axial velocity is shown in Figure 8. By observing Figure 8, the fluid velocity is decreasing as the Hartmann number is increasing. Further, as $H \rightarrow 0$, the Hartmann number loses its properties and behaves like a normal blood flow without any magnetic field applied to it. We have concluded that the application of magnetic field reduces the speed of blood flow and that meets the results of Ponalagusamy and Tamil Selvi [25], also indicating that the 


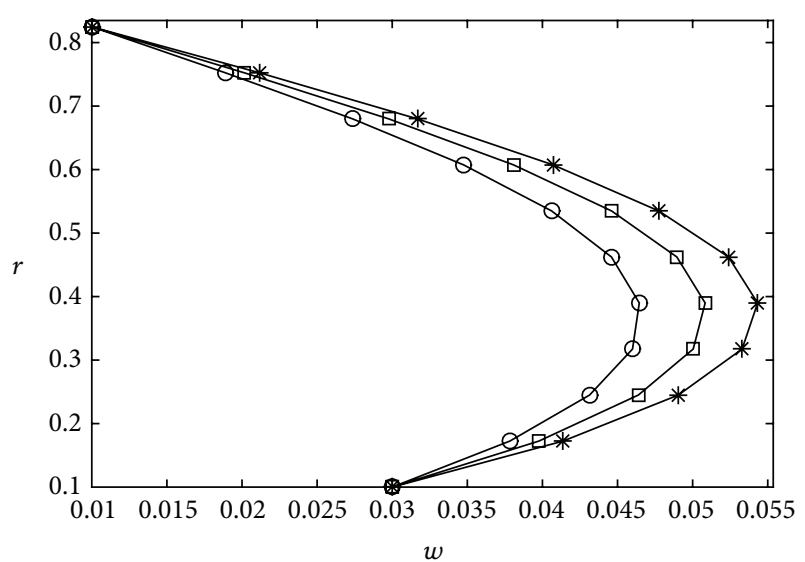

$$
\begin{aligned}
& -\beta=5 \\
& \square \beta=6 \\
& * \beta=7
\end{aligned}
$$

FIGURE 6: Variation of axial velocity $w$ with respect to $\beta$ when $n=5$, $\epsilon=0.2, \zeta=0, H=1, v=0.01, g=0.03$, and $R_{c}=0.1$.

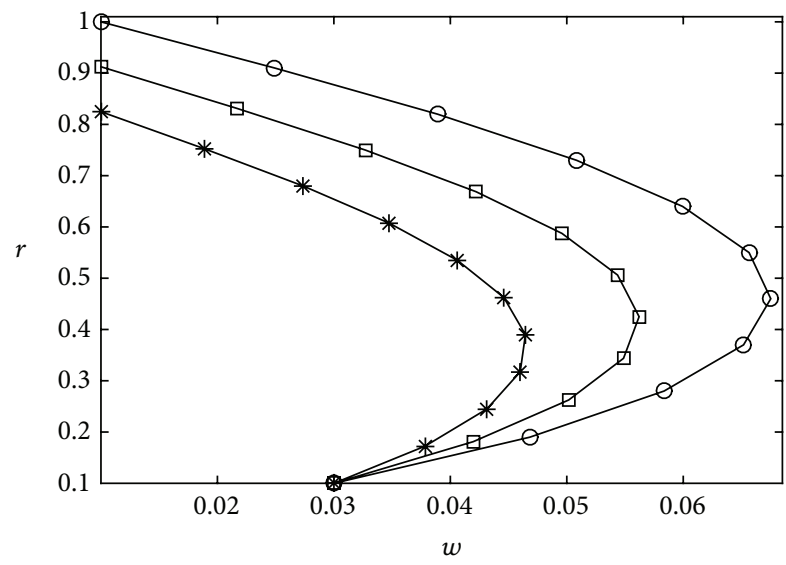

$$
\begin{aligned}
& -\epsilon=0.0 \\
& \square-\epsilon=0.1 \\
& \rightarrow-\epsilon=0.2
\end{aligned}
$$

FIgURE 7: Variation of axial velocity $w$ with respect to $\epsilon$ when $n=5$, $\zeta=0, \beta=5, H=1, v=0.01, g=0.03$, and $R_{c}=0.1$.

wall shear stress increases when we increase the Hartmann number. The influence of catheter radius $R_{c}$ on the axial velocity is directly proportional as shown in Figure 9. From the above result, we understand that as the catheter radius increases, the annular region gets narrowed, which leads to the rise in the obstruction to the flow.

The axial velocity of the blood is high in case of high slip velocity $v$ or catheter velocity $g$; it is observed that the slip velocity at the boundary facilitates the fluid flow and the same goes for the moving catheter, Figures 10 and 11.

The shear stress at the wall is a significant physiological parameter to be considered in the blood flow study. Precise predictions of the distribution of the shear stress at the wall are particularly useful in assimilating the effect of blood flow in arteries in general. The shear stress at the wall is calculated

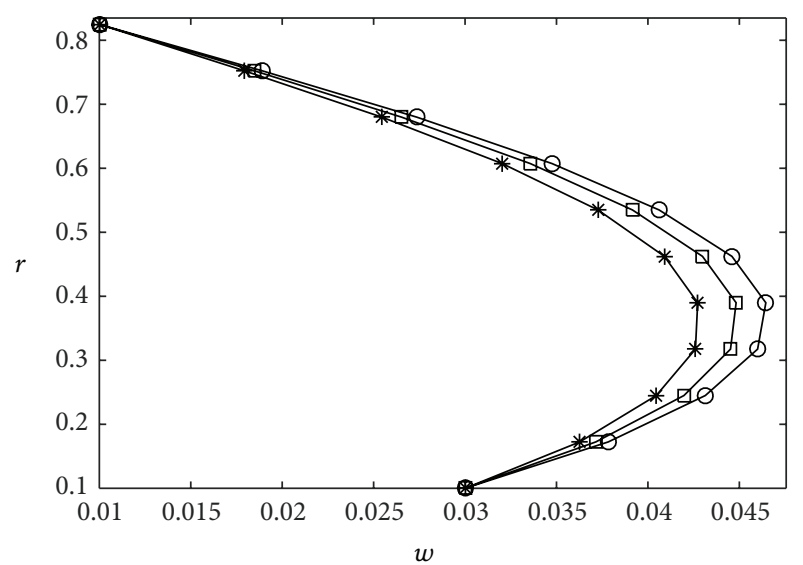

$$
\begin{aligned}
& -H=1.0 \\
& \square H=1.5 \\
& \text { * } H=20
\end{aligned}
$$

FIGURE 8: Variation of axial velocity $w$ with respect to $H$ when $n=5$, $\zeta=0, \beta=5, \epsilon=0.2, v=0.01, g=0.03$, and $R_{c}=0.1$.

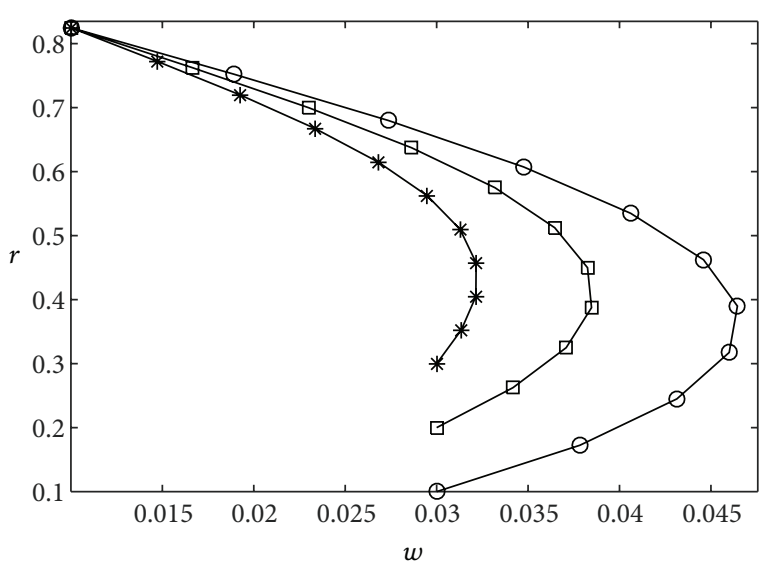

$$
\begin{aligned}
& \because R_{c}=0.1 \\
& \square-R_{c}=0.2 \\
& -R_{c}=0.3
\end{aligned}
$$

FigURE 9: Variation of axial velocity $w$ with respect to $R_{c}$ when $n=5$, $\zeta=0, \beta=5, \epsilon=0.2, v=0.01, g=0.03$, and $H=1$.

at the maximum height of the stenosis. When we increase the height of the stenosis $\epsilon$, the wall shear stress increases, Figure 15. Furthermore, it is observed that the shear stress along the wall is reaching the maximum at the throat of the stenosis.

The effect of the Hartmann number $H$ on the shear stress is shown in Figure 16. As observed from Figure 12, the shear stress at the wall is independent of the shape parameter $n$ as pointed out by Ponalagusamy and Tamil Selvi [25]. The variation of the shear stress at the wall as the tapered parameter increases is depicted in Figure 13. Here, the shear stress at the wall is more significant in the untapered artery compared with the tapered artery which is diverging. Further, the converging tapered artery possesses higher wall 


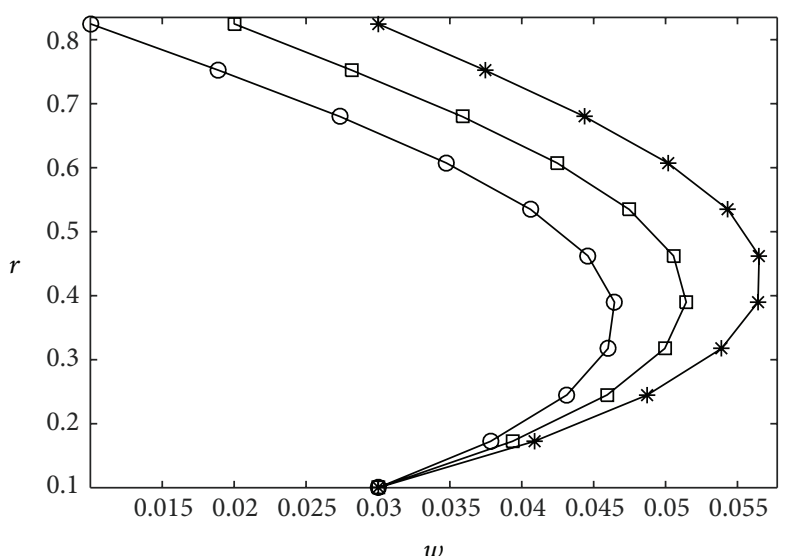

$$
\begin{aligned}
& \because v=0.01 \\
& \square \quad v=0.02 \\
& * \quad v=0.03
\end{aligned}
$$

FIGURE 10: Variation of axial velocity $w$ with respect to $v$ when $n=5$, $\zeta=0, \beta=5, \epsilon=0.2, R_{c}=0.1, g=0.03$, and $H=1$.

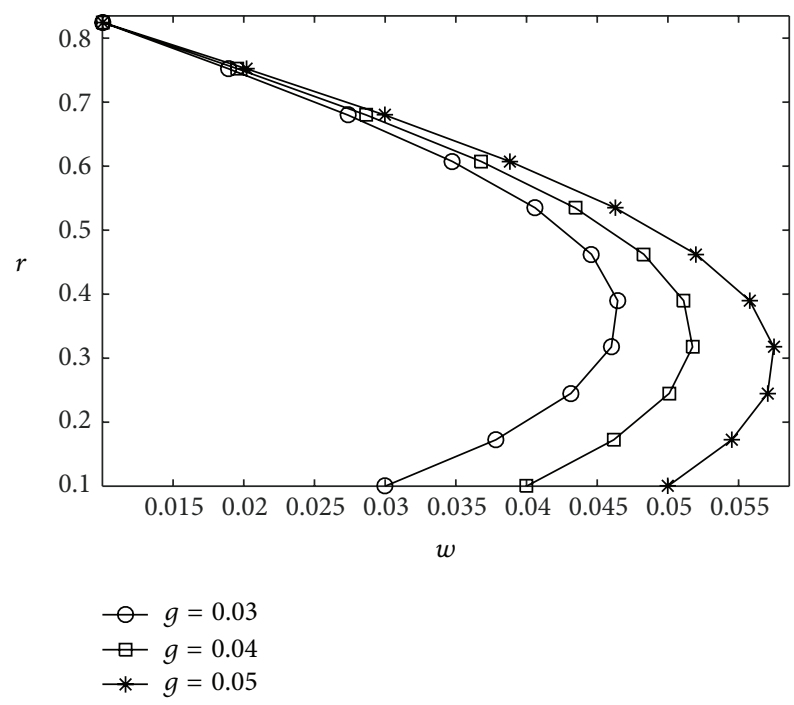

FIGURE 11: Variation of axial velocity $w$ with respect to $g$ when $n=5$, $\zeta=0, \beta=5, \epsilon=0.2, v=0.01, R_{c}=0.1$, and $H=1$.

shear stress than the untapered and tapered artery which is diverging.

The slip velocity at the wall of the stenosed artery and the moving catheter significantly influence the shear stress at the wall, which is noticed from Figures 18 and 19. Here, it is observed that gain in slip velocity (resp., moving catheter) reduces the shear stress at the wall. The influence of catheter radius on shear stress at the wall is shown in Figure 17. The increasing catheter radius narrows down the lumen of the artery thus resulting in the higher values of shear stress at the wall. As couple-stress fluid parameter $\beta$ increases, the shear stress at the wall decreases. This behavior is shown in Figure 14. It is observed that the theoretical distribution of shear stress along the wall reaches a maximum at throat of the stenosis and then rapidly decreases in the diverging section.

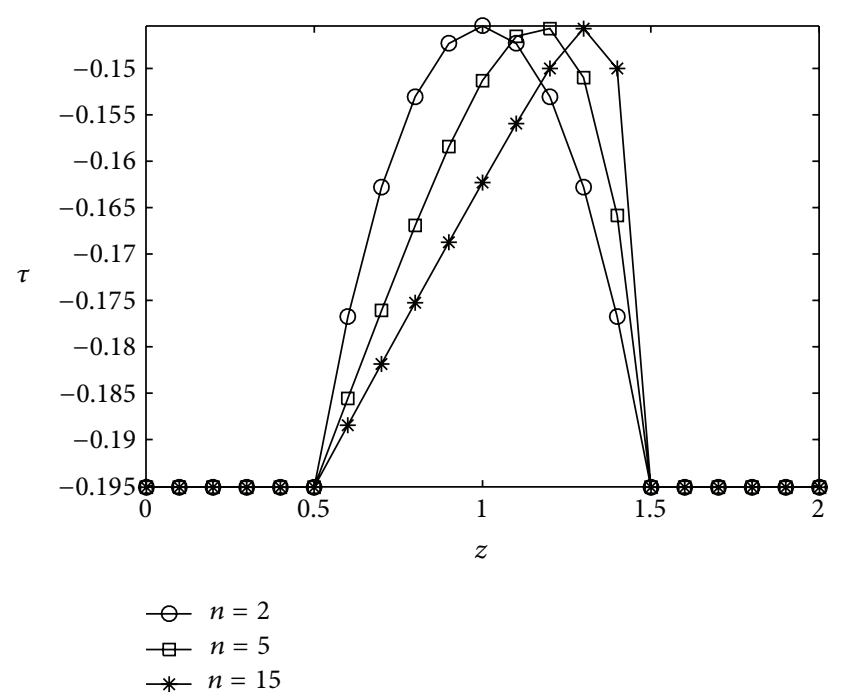

FIGURE 12: Variation of wall shear stress $w$ with respect to $n$ when $\zeta=0, \epsilon=0.2, \beta=5, H=1, v=0.01, g=0.03$, and $R_{c}=0.1$.

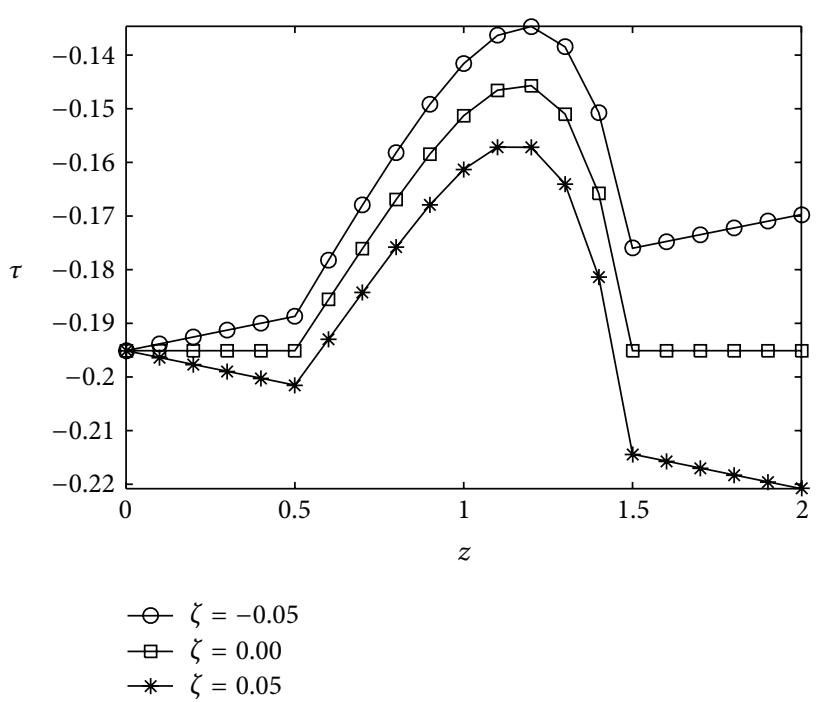

FIGURE 13: Variation of wall shear stress $w$ with respect to $\zeta$ when $n=5, \epsilon=0.2, \beta=5, H=1, v=0.01, g=0.03$, and $R_{c}=0.1$.

This behavior is the same in terms of the change in both the radius of the catheter $R_{c}$ and the couple-stress fluid parameter $\beta$ whose behavior is depicted graphically in Figures 17 and 14, respectively.

\section{Conclusion}

A mathematical model has been built to discuss the flow of blood through a catheterized asymmetric tapered stenosed artery with slip velocity at the stenosed wall and a moving catheter. Closed form solution is obtained and the effects of various geometric, fluid parameters and magnetic field on the axial velocity of the blood and the shear stress at the wall are studied. 


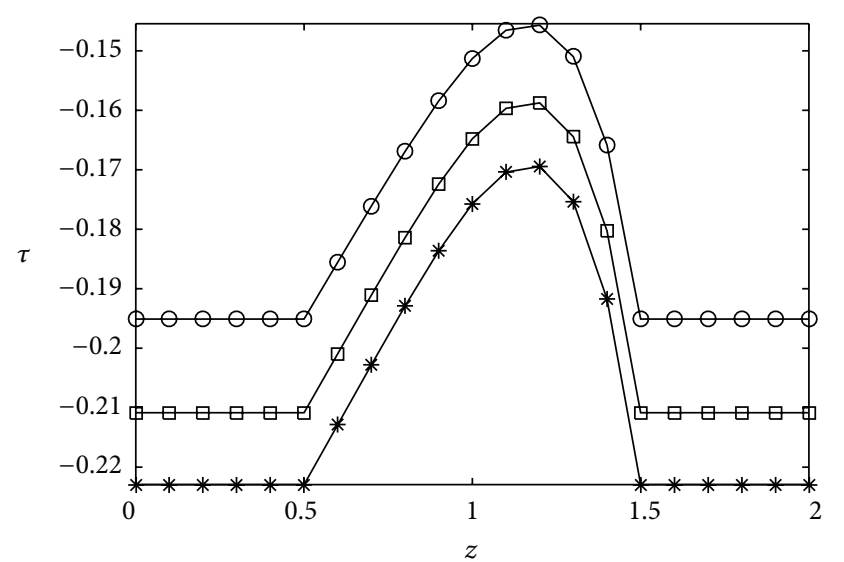

$$
\begin{aligned}
& -\beta=5 \\
& \square \beta=6 \\
& * \beta=7
\end{aligned}
$$

FIGURE 14: Variation of wall shear stress $w$ with respect to $\beta$ when $n=5, \epsilon=0.2, \zeta=0, H=1, v=0.01, g=0.03$, and $R_{c}=0.1$.

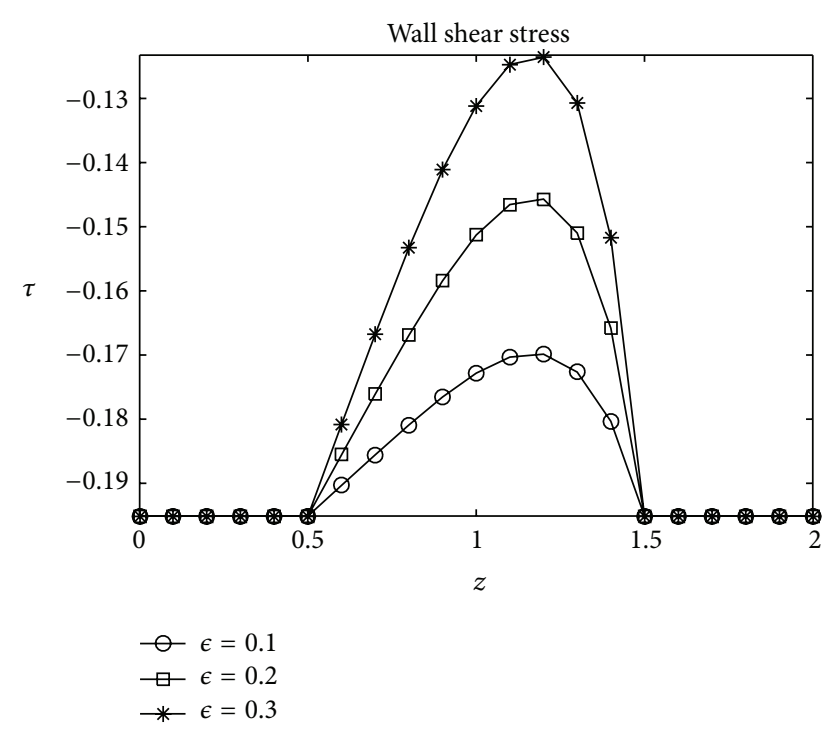

FIGURE 15: Variation of wall shear stress $w$ with respect to $\epsilon$ when $n=5, \zeta=0, \beta=5, H=1, v=0.01, g=0.03$, and $R_{c}=0.1$.

There is special importance of couple-stress fluids compared to the Newtonian fluids because of their wide existence such as oil, blood, and polymeric solutions. In view of what is mentioned above, an analytic approach was followed to solve the mathematical model of blood flow through stenosed tapered artery under the assumption of mild stenosis. The resultant observations are summarized as follows:

(i) As the height and the stenosis length are increasing, the obstruction to the flow of blood is increasing.

(ii) Converging tapered artery has more shear stress at the wall than the nontapered and the diverging tapered artery.

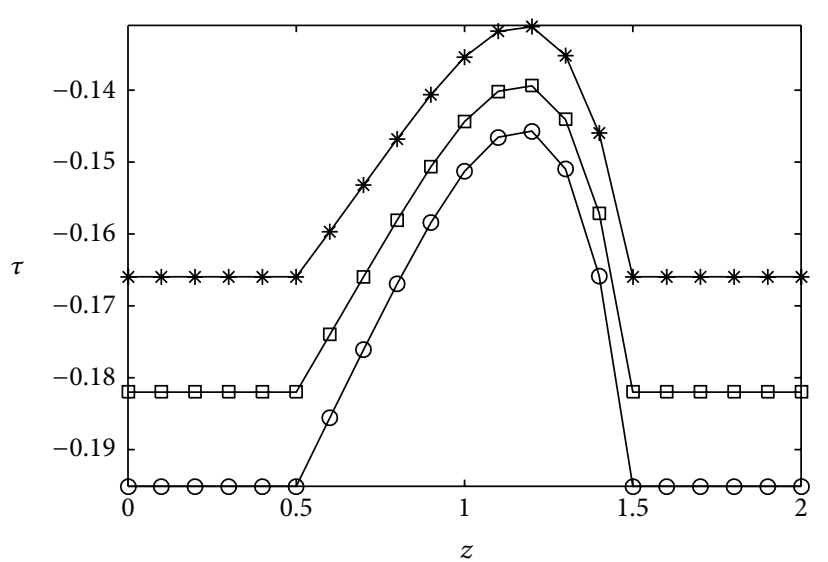

$$
\begin{aligned}
& -H=1.0 \\
& \text { - } H=1.5 \\
& \text { * } H=2.0
\end{aligned}
$$

FIGURE 16: Variation of wall shear stress $w$ with respect to $H$ when $n=5, \zeta=0, \beta=5, \epsilon=0.2, v=0.01, g=0.03$, and $R_{c}=0.1$.

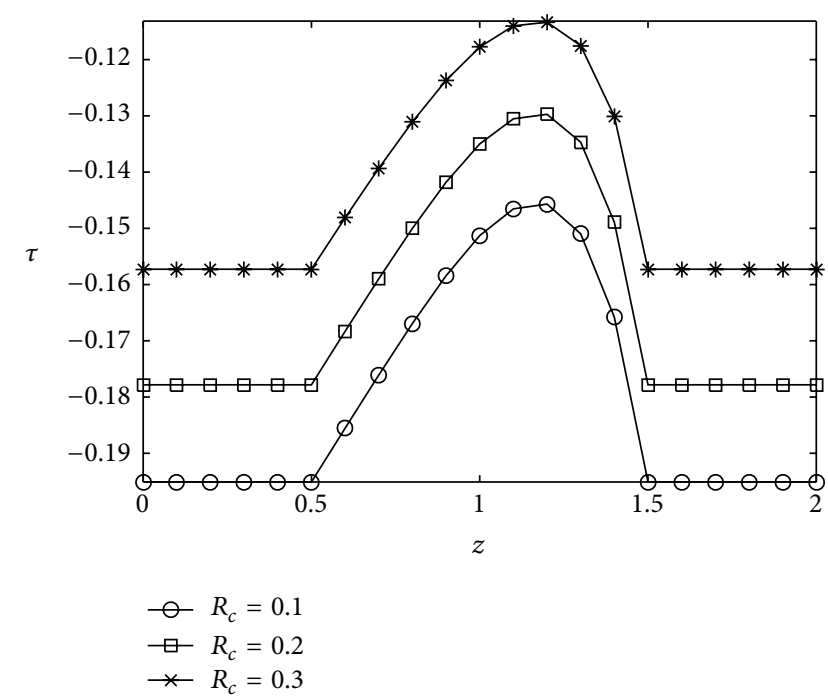

FIGURE 17: Variation of wall shear stress $w$ with respect to $R_{c}$ when $n=5, \zeta=0, \beta=5, \epsilon=0.2, v=0.01, g=0.03$, and $H=1$.

(iii) Diverging tapered artery has least shear stress at the wall.

(iv) The increment in the Hartmann number enhances the blood velocity and wall shear stress.

(v) The axial velocity is decreasing while the couple-stress fluid parameters $\beta$ and the height of the stenosis $\epsilon$ are increasing.

(vi) Three different values of the slip velocity and the velocity of the catheter at the arterial boundary and the catheter wall are considered; they are showing a significant influence on the shear stress at the wall and the axial velocity.

The modeling and simulation of the above phenomena are very realistic and are expected to be very useful in 


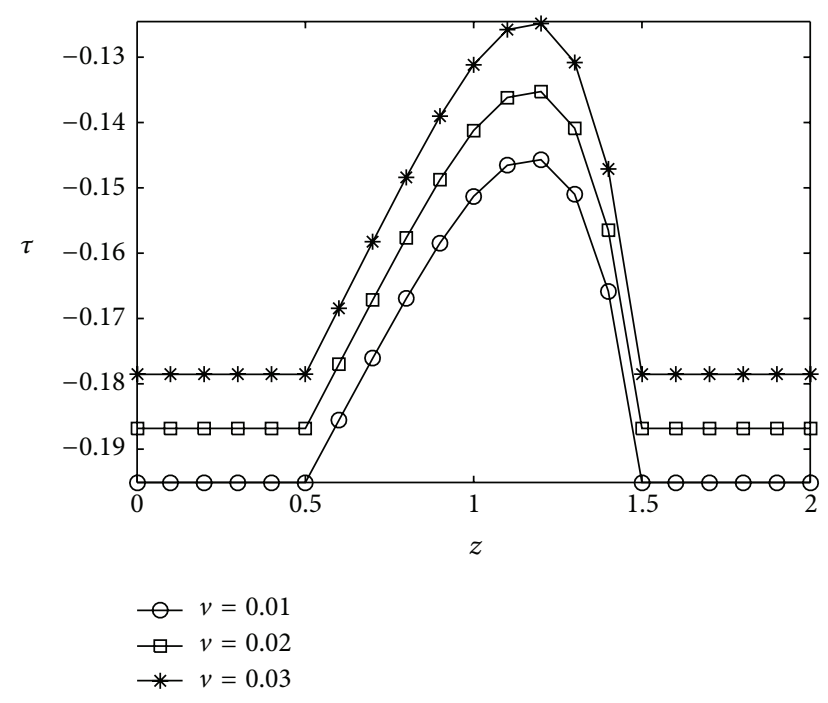

FIGURE 18: Variation of wall shear stress $w$ with respect to $v$ when $n=5, \zeta=0, \beta=5, \epsilon=0.2, R_{c}=0.1, g=0.03$, and $H=1$.

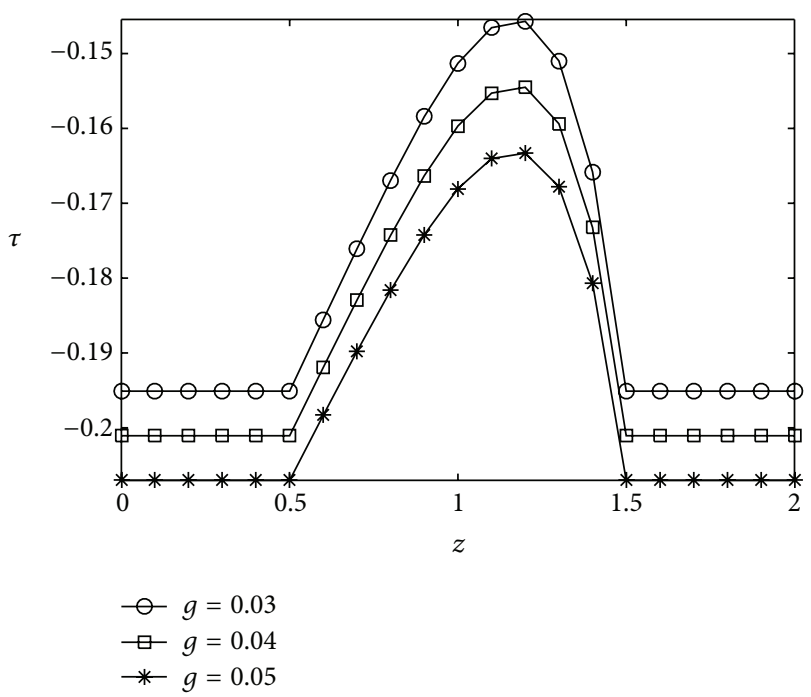

FIGURE 19: Variation of wall shear stress $w$ with respect to $g$ when $n=5, \zeta=0, \beta=5, \epsilon=0.2, v=0.01, R_{c}=0.1$, and $H=1$.

predicting the behavior of physiological parameters in the diagnosis of various arterial diseases.

\section{Symbols}

$\begin{array}{ll}\text { B: } & \text { Magnetic flux intensity } \\ B_{0}: & \text { External transverse } \\ & \text { magnetic field } \\ & \text { Constants of integration } \\ c_{i}, i=1, \ldots, 4: & \text { Location of the stenosis } \\ d: & \text { Defined constants } \\ d_{i}=-c_{i} / k_{s}, i=1, \ldots, 4 & \text { Electric field intensity } \\ \mathbf{E}: & \text { Body force } \\ \mathbf{f}: & \text { Electromagnetic force } \\ \mathbf{F}_{m}: & \end{array}$

$g:$

$H=B \sqrt{R_{0}^{2} \sigma / \mu}:$
$I_{0}:$

$\mathrm{J}:$

$K_{0}$ :

l:

$L_{0}$ :

M:

$n_{1}=\sqrt{\left(\beta^{2}+\sqrt{\beta^{4}-4 \beta^{2} H^{2}}\right) / 2}:$

$n_{2}=\sqrt{\left(\beta^{2}-\sqrt{\beta^{4}-4 \beta^{2} H^{2}}\right) / 2}$ :

$p:$

$Q:$

$R_{c}$ :

$\operatorname{Re}=\rho u_{0} R_{0} / \mu$ :

$R(z)$ :

$u$ :

V:

$v:$

$w:$

$w_{0}$ :

$\beta=R_{0} \sqrt{\mu / \eta}$ :

$\Delta=\partial^{2} / \partial r^{2}+(1 / r)(\partial / \partial r):$

$\epsilon$ :

$\eta, \eta^{\prime}:$

$\Gamma=L_{0} / d$ :

$\gamma=L_{0} / L_{1}$ :

$R_{f}$ :

$\lambda, \mu:$

$\omega=\eta^{\prime} / \eta$ :

$\phi:$

$\rho:$

$\sigma:$

$\tau:$

$\tau_{r z}:$

$\tau_{r z}^{S}$

$\xi=R_{0} / L_{0}:$

$\zeta$ :
Velocity of the moving catheter

Hartmann number

Modified Bessel function of the first kind and order

zero

Current density

Modified Bessel function of the second kind and order zero

Body couple moment

Stenosis length

Couple-stress tensor

: Defined constant

Defined constant

Pressure

Volumetric flow rate

Radius of the

nonconstricted region

Reynolds number

Radius of the tube

Radial velocity

Velocity vector

Slip velocity

Axial velocity

Typical axial velocity

Couple-stress fluid

parameter

Laplacian operator

Maximum height of the stenosis

Couple-stress coefficients

Defined constant

Defined constant

Resistance to the flow

Viscosity coefficients

Parameter associated with the couple-stress fluid

Tapering angle

Density of the fluid

Electrical conductivity

Shear stress tensor

Shear stress

Skew-symmetric part of shear stress

Symmetric part of shear

stress

Defined constant

Tapering parameter.

\section{Conflict of Interests}

The authors declare that there is no conflict of interests regarding the publication of this paper. 


\section{References}

[1] R. C. Diggery and D. T. Grint, Catheters: Types, Applications and Potential Complications, Nova Science, 2012.

[2] D. Filgueiras-Rama, A. Estrada, J. Shachar et al., "Remote magnetic navigation for accurate, real-time catheter positioning and ablation in cardiac electrophysiology procedures," Journal of Visualized Experiments, no. 74, Article ID e3658, 2013.

[3] B. Mols, "Magnetic-field navigation for catheters," TU Delft Outlook, University Magazine, 2004.

[4] V. K. Stokes, Theories of Fluids with Microstructure: An Introduction, Springer, Berlin, Germany, 1984.

[5] A. C. Eringen, Theory of Micropolar Fluids, no. RR-27, Purdue University School of Aeronautics and Astronautics, West Lafayette, Ind, USA, 1965.

[6] S. C. Cowin, "The theory of polar fluids," Advances in Applied Mechanics, vol. 14, pp. 82-279, 1974.

[7] P. Chaturani and V. S. Upadhya, "Pulsatile flow of a couple stress fluid through circular tubes with applications to blood flow," Biorheology, vol. 15, no. 3-4, pp. 193-201, 1978.

[8] P. Chaturani and V. P. Rathod, "A critical study of Poiseuille flow of couple stress fluid with applications to blood flow," Biorheology, vol. 18, no. 2, pp. 235-244, 1981.

[9] A. Kollin, "Electromagnetic flowmeter: Principle of method and its application to blood flow measurement," Proceedings of the Society for Experimental Biology and Medicine, vol. 35, pp. 235244, 1936.

[10] E. M. Korchevskii and L. S. Marochnik, "Magnetohydrodynamic version of movement of blood," Biophysics, vol. 10, no. 2, pp. 411-413, 1965.

[11] A. R. Rao and K. S. Deshikachar, "Physiological type flow in a circular pipe in the presence of a transverse magnetic field," Journal of the Indian Institute of Science, vol. 68, pp. 247-260, 1988.

[12] V. A. Vardanyan, "Effect of a magnetic field on blood flow," Biofizika, vol. 18, no. 3, pp. 491-496, 1973.

[13] V. P. Rathod and Gayatri, "Effect of magnetic field on two layer model of pulsatile blood flow with micro-organisms," Bulletin of Pure and Applied Sciences E. Mathematics and Statistics, vol. 19, no. 1, pp. 1-13, 2000.

[14] D. S. Sankar and K. Hemalatha, "Pulsatile flow of HerschelBulkley fluid through catheterized arteries-a mathematical model," Applied Mathematical Modelling, vol. 31, no. 8, pp. 14971517, 2007.

[15] D. Srinivasacharya and D. Srikanth, "Effect of couple stresses on the pulsatile flow through a constricted annulus," Comptes Rendus Mécanique, vol. 336, no. 11-12, pp. 820-827, 2008.

[16] D. N. Riahi, R. Roy, and S. Cavazos, "On arterial blood flow in the presence of an overlapping stenosis," Mathematical and Computer Modelling, vol. 54, no. 11-12, pp. 2999-3006, 2011.

[17] V. P. Srivastava and S. Mishra, "Non-Newtonian arterial blood flow through an overlapping stenosis," Applications and Applied Mathematics, vol. 5, no. 1, pp. 225-238, 2010.

[18] S. Chakravarty and P. K. Mandal, "Mathematical modelling of blood flow through an overlapping arterial stenosis," Mathematical and Computer Modelling, vol. 19, no. 1, pp. 59-70, 1994.

[19] V. Vand, "Viscosity of solutions and suspensions. I. Theory," The Journal of Physical Chemistry, vol. 52, no. 2, pp. 277-299, 1948.

[20] L. Bennett, "Red cell slip at a wall in vitro," Science, vol. 155, no. 3769, pp. 1554-1556, 1967.
[21] Y. Nubar, "Blood flow, slip, and viscometry," Biophysical Journal, vol. 11, no. 3, pp. 252-264, 1971.

[22] P. Chaturani and D. Biswas, "A comparative study of poiseuille flow of a polar fluid under various boundary conditions with applications to blood flow," Rheologica Acta, vol. 23, no. 4, pp. 435-445, 1984.

[23] R. Ponalagusamy, "Blood flow through an artery with mild stenosis: a two-layered model, different shapes of stenoses and slip velocity at the wall," Journal of Applied Sciences, vol. 7, no. 7, pp. 1071-1077, 2007.

[24] B. K. Mishra and S. S. Shekhawat, "Magnetic effect on blood flow through a tapered artery with stenosis," Acta Ciencia Indica, vol. 1029, pp. 295-301, 2007.

[25] R. Ponalagusamy and R. Tamil Selvi, "Blood flow in stenosed arteries with radially variable viscosity, peripheral plasma layer thickness and magnetic field," Meccanica, vol. 48, no. 10, pp. 2427-2438, 2013.

[26] D. Biswas and U. S. Chakraborty, "Pulsatile blood flow through a catheterized artery with an axially nonsymmetrical stenosis," Applied Mathematical Sciences, vol. 4, pp. 2865-2880, 2010. 


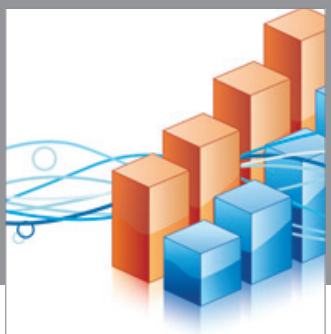

Advances in

Operations Research

vatem alat4

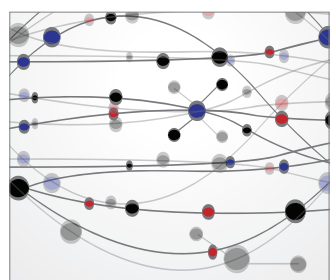

\section{The Scientific} World Journal
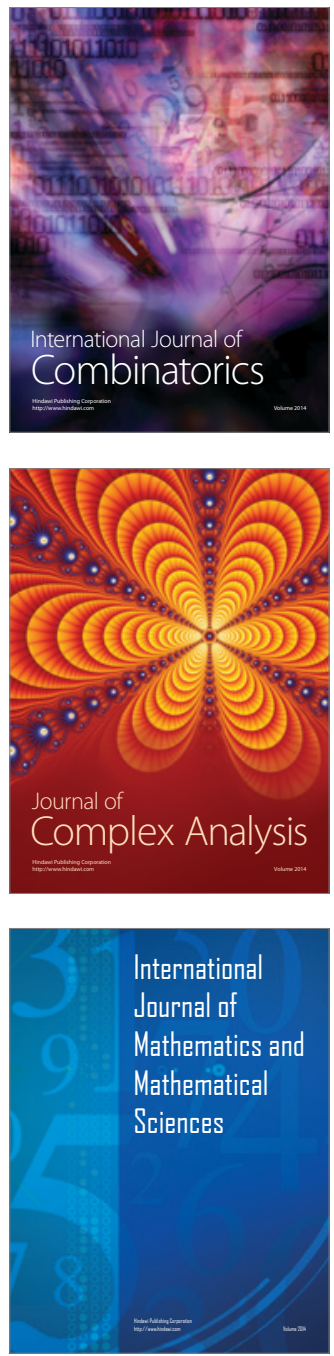
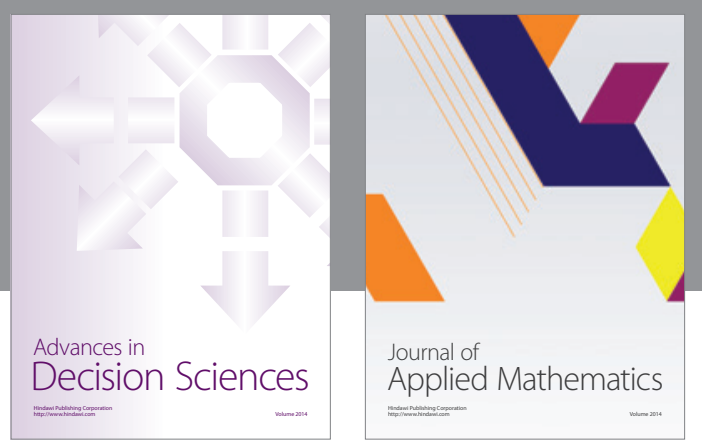

Algebra

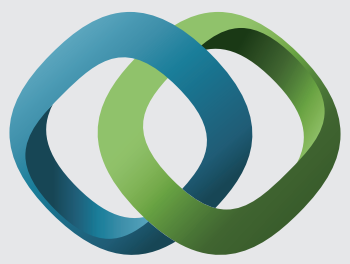

\section{Hindawi}

Submit your manuscripts at

http://www.hindawi.com
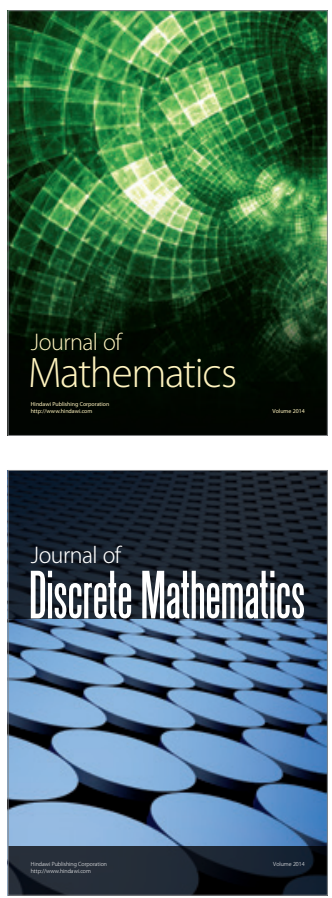

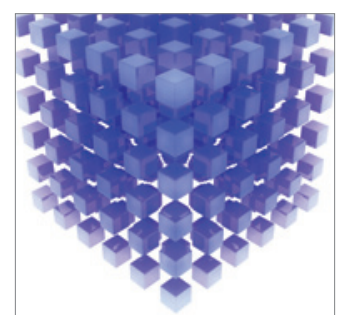

Mathematical Problems in Engineering
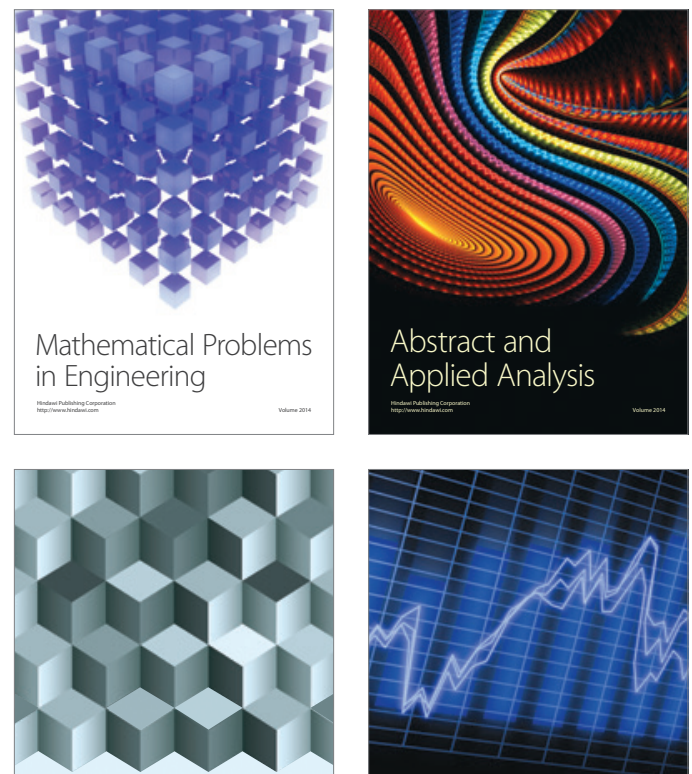

Journal of

Function Spaces

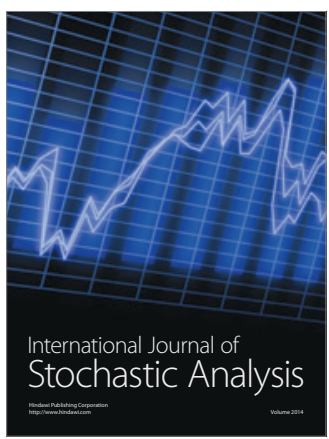

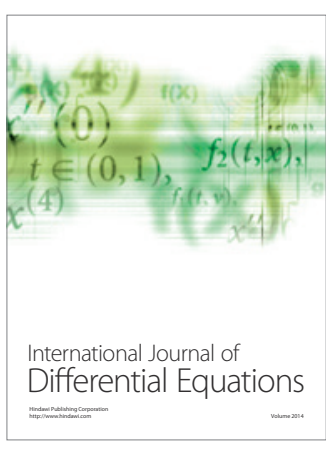
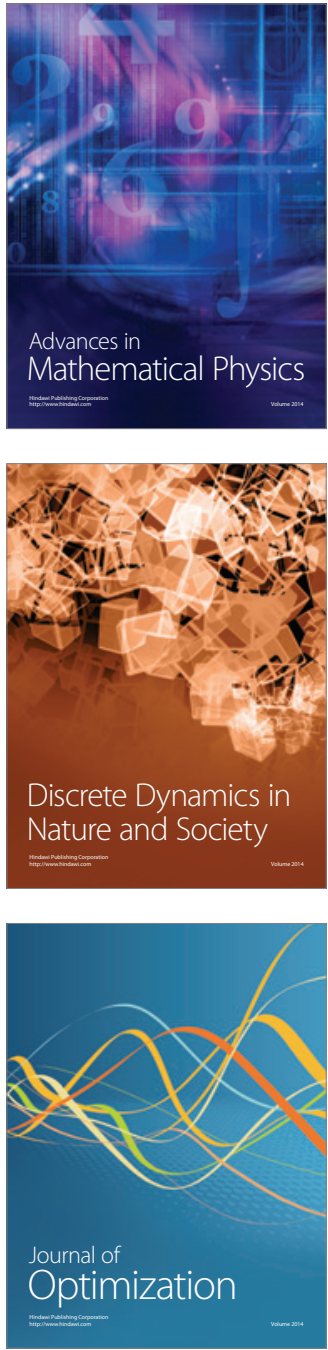